OPEN ACCESS

Edited by:

Robert Sinden,

Imperial College London,

United Kingdom

Reviewed by:

Jingwen Wang,

Yale University, United States

Gabriele Pradel,

RWTH Aachen University, Germany

*Correspondence:

Shirley Luckhart

sluckhart@uidaho.edu

Specialty section:

This article was submitted to

Parasite and Host,

a section of the journal

Frontiers in Cellular and

Infection Microbiology

Received: 09 August 2020 Accepted: 13 November 2020 Published: 11 December 2020

Citation:

Luckhart S and Riehle MA (2020) Midgut Mitochondrial Function as a

Gatekeeper for Malaria Parasite Infection and Development in the Mosquito Host. Front. Cell. Infect. Microbiol. 10:593159. doi: 10.3389/fcimb.2020.593159

\section{Midgut Mitochondrial Function as a Gatekeeper for Malaria Parasite Infection and Development in the Mosquito Host}

\author{
Shirley Luckhart ${ }^{1,2 *}$ and Michael A. Riehle ${ }^{3}$ \\ ${ }^{1}$ Department of Entomology, Plant Pathology and Nematology, University of Idaho, Moscow, ID, United States, \\ ${ }^{2}$ Department of Biological Sciences, University of Idaho, Moscow, ID, United States, ${ }^{3}$ Department of Entomology, University \\ of Arizona, Tucson, AZ, United States
}

Across diverse organisms, various physiologies are profoundly regulated by mitochondrial function, which is defined by mitochondrial fusion, biogenesis, oxidative phosphorylation (OXPHOS), and mitophagy. Based on our data and significant published studies from Caenorhabditis elegans, Drosophila melanogaster and mammals, we propose that midgut mitochondria control midgut health and the health of other tissues in vector mosquitoes. Specifically, we argue that trade-offs among resistance to infection, metabolism, lifespan, and reproduction in vector mosquitoes are fundamentally controlled both locally and systemically by midgut mitochondrial function.

Keywords: mosquito, malaria, Anopheles, Plasmodium, mitochondria, midgut, resistance, immunity

\section{INTRODUCTION}

Across diverse organisms, physiology is profoundly regulated by mitochondrial function, which is defined by the sum of mitochondrial fusion, biogenesis, oxidative phosphorylation (OXPHOS) and mitophagy (Zhu et al., 2013; Yu et al., 2020). Based on significant work from Caenorhabditis elegans (Chikka et al., 2016; Kwon et al., 2018) and Drosophila melanogaster (Miguel-Aliaga et al., 2018), it is likely that mitochondrial control of tissue health - and gut health in particular-is conserved across the Ecdysozoa. Importantly, the balance of mitochondrial function and dysfunction, particularly in the midgut, is highly relevant to mosquito vectors of human pathogens (Luckhart and Riehle, 2017). We hypothesize that mitochondrial activity in the midgut, the initial interface between the mosquito and Plasmodium parasites, dictates the balance of pathogen susceptibility and the life history traits essential

Abbreviations: 20E, 20-hydroxyecdysone; Akt, IIS kinase, also known as protein kinase B; AMPK, 5' adenosine monophosphate-activated protein kinase; Atg, autophagy; ERK, extracellular signal-regulated kinase; ETC, electron transport chain; FOXO, class O forkhead box transcription factors; IGF1, insulin-like growth factor 1; IIS, insulin/insulinlike growth factor signaling; ILP, insulin-like peptide; IRS, insulin receptor substrate; MEK, mitogen-activated protein kinase kinase; MSC, midgut stem cells; mTOR, mechanistic target of rapamycin; NADH, reduced form of nicotinamide adenine dinucleotide; NF- $\mathrm{KB}$, nuclear factor-kappa B; NO, nitric oxide; NTG, non-transgenic; $\mathrm{OEH}$, ovarian ecdysteroidogenic hormone; OXPHOS, oxidative phosphorylation; p38 MAPK, p38 mitogen-activated protein kinase; p70S6K, p70 S6 kinase; PGC-1, peroxisome proliferator-activated receptor gamma coactivator-1; PI-3K, phosphatidylinositol-3-kinase; PINK1, PTEN-induced kinase 1; PTEN, IIS protein, also known as phosphatase and tensin homolog; ROS, reactive oxygen species; TG, transgenic. 
for ensuring pathogen transmission. Further, the regulation of midgut mitochondrial activity is regulated in a large part through the insulin/insulin-like growth factor signaling (IIS) cascade. In this review, we will discuss data that support the inference that trade-offs among resistance to infection, lifespan and reproduction in mosquito vectors of malaria parasites are fundamentally controlled both locally and systemically by midgut mitochondrial function via IIS. While this mini-review will focus on mitochondrial activity in the mosquito gut, due to the central regulatory role of the gut and its unique interaction with pathogens in the bloodmeal, it is likely that mitochondrial activity in diverse tissues also has important roles in regulating the physiologies described below.

\section{INSULIN/INSULIN-LIKE GROWTH FACTOR SIGNALING CONTROLS MITOCHONDRIAL FUNCTION TO ALTER DIVERSE PHYSIOLOGIES IMPACTING VECTORIAL CAPACITY}

IIS regulates mitochondrial function across a wide range of organisms and, in this capacity, contributes to control of longevity, host immunity, cellular metabolism and the response to stress in mammals and in the model organisms D. melanogaster and C. elegans. We and others have demonstrated that IIS in adult female mosquitoes regulates egg production, longevity, defenses against infection, metabolism and the host stress response (Graf et al., 1997; Riehle and Brown, 1999; Riehle and Brown, 2002; Riehle and Brown, 2003; Lim et al., 2005; Kang et al., 2007; Roy et al., 2007; Brown et al., 2008; Sim and Denlinger, 2008; Arik et al., 2009; Corby-Harris et al., 2010; Horton et al., 2010; Gulia-Nuss et al., 2011; Marquez et al., 2011; Surachetpong et al., 2011; Pakpour et al., 2012; Hauck et al., 2013; Luckhart et al., 2013; Drexler et al., 2014; Arik et al., 2015; Pietri et al., 2016; Nuss and Brown, 2018). Further, substantial data indicate that IIS-dependent phenotypes are mediated through changes in mitochondrial function (Figure 1) in model invertebrates, mosquitoes and in mammals (Tóth et al., 2008; Cheng et al., 2010; Tiefenbock et al., 2010; Luckhart et al., 2013; Sadagurski and White, 2013; Drexler et al., 2014; Mukherjee et al., 2014; Pietri et al., 2016; Chaudhari and Kipreos, 2017; Ruegsegger et al., 2018; Wang et al., 2019; Wardelmann et al., 2019; Charmpilas and Tavernarakis, 2020; Sheard et al., 2020). Specifically, perturbations of both the IIS activator Akt and the inhibitor PTEN in the midgut of Anopheles stephensi led to profound changes in midgut mitochondrial number, quality and function as discussed below (Luckhart et al., 2013; Hauck et al., 2013).

\section{MITOCHONDRIAL FUNCTION IS EVOLUTIONARILY CONSERVED AND REGULATED BY MITOCHONDRIAL QUALITY CONTROL}

Mitochondrial biogenesis or the generation of new mitochondria and elimination of damaged mitochondria via mitophagy, a form of organelle-specific autophagy, control mitochondrial function and have profound effects on health and disease in diverse organisms (Palikaras et al., 2018). In particular, the balance between mitochondrial biogenesis and mitophagy is highly responsive to the host environment, including changes in cellular metabolism and energy status and activation of immunity to infection, to ensure tissue and organismal homeostasis (Youle and van der Bliek, 2012; Ma et al., 2020).

In general, mitochondrial damage from oxidative stress, stressinduced changes in energy status and xenobiotic insults can initiate mitochondrial fission or the separation of compromised mitochondria for removal by mitophagy (Elgass et al., 2012). The genomes of A. stephensi and Anopheles gambiae encode autophagy machinery orthologs for Parkin, PINK1, more than a dozen autophagy-related (Atg) genes and two vacuolar protein sorting genes. In brief, damaged mitochondria are targeted for the aggregation of PINK1, which recruits the E3 ubiquitin ligase Parkin to activate Atg proteins to form the autophagosome. Activators of autophagy include mitochondrial reactive oxygen species (ROS) as well as signaling through ERK-dependent and p38 MAPK and AMP-activated protein kinase (AMPK; Huang et al., 2011; Levine et al., 2011; Corona Velazquez and Jackson, 2018). Autophagy is inhibited by signaling through the PI-3K/Akt branch of the IIS cascade, signaling that also controls mitochondrial biogenesis (Huang et al., 2011; Levine et al., 2011; Denton et al., 2012; Manning and Toker, 2017). Specifically, when FOXO is excluded from the nucleus by PI-3K/Akt signaling, PPAR gamma coactivator-1 alpha (PGC-1), a key mediator of mitochondrial biogenesis, is not activated (Cheng et al., 2009). Conversely, overexpression of the IIS inhibitor PTEN, which results in FOXO translocation into the nucleus, induces PGC-1 activity (Garcia-Cao et al., 2012). In D. melanogaster, FOXO-induced PGC-1 increased levels and activity of electron transport chain (ETC) complexes I, II, and IV, indicating that enhanced functioning of the ETC is positively associated with biogenesis (Rera et al., 2011). Increased midgut mitochondrial biogenesis in D. melanogaster resulted in improved midgut epithelial integrity, and we have associated this biological dynamic in A. stephensi with reduced development of the human malaria parasite Plasmodium falciparum in the absence of enhanced NF- $\kappa \mathrm{B}$-dependent immune gene expression (Rera et al., 2011; Pakpour et al., 2012; Hauck et al., 2013; Pakpour et al., 2013). Mitochondrial biogenesis, which is activated by NO-dependent guanylate cyclase as well as by AMPK signaling, was hyperactivated and out of balance with mitophagy in A. stephensi engineered to overexpress activated Akt in the midgut epithelium (Nisoli et al., 2003; Nisoli et al., 2004; Nisoli et al., 2005; Fernandez-Marcos and Auwerx, 2011; Luckhart et al., 2013). While Akt-dependent mitochondrial dysfunction was deleterious to A. stephensi, it also conferred extreme resistance to $P$. falciparum infection (CorbyHarris et al., 2010; Luckhart et al., 2013).

\section{MITOCHONDRIAL FUNCTION CONTROLS EPITHELIAL BARRIER INTEGRITY AND STEM CELL ACTIVITY IN THE GUT}

An analysis of mitochondrially controlled phenotypes is informative for the idea that mitochondria are master regulators 


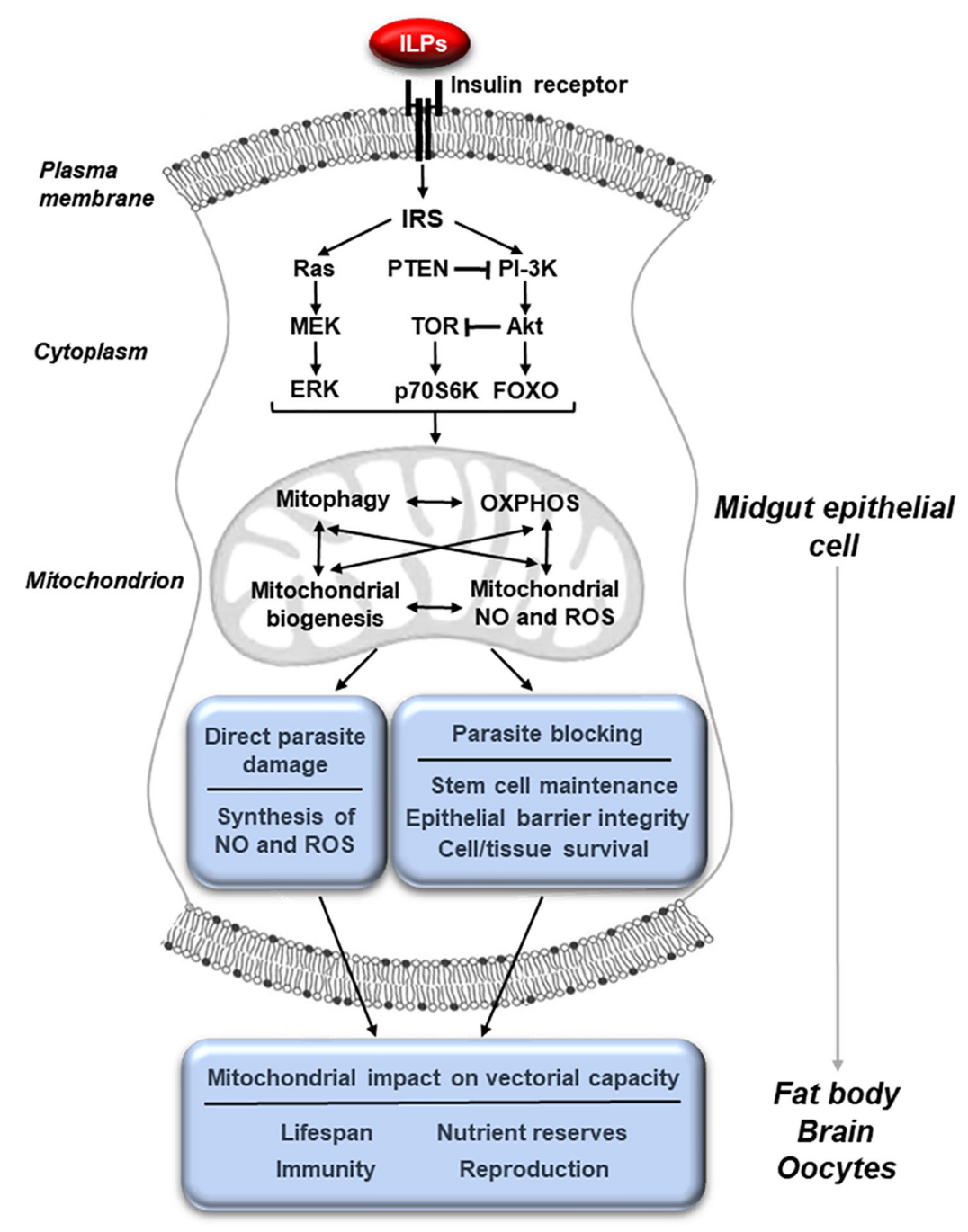

FIGURE 1 | Midgut mitochondrial function, activated through IIS, controls physiologies that govern vectorial capacity. The binding of insulin-like peptides (ILPS) to the insulin receptor at the membrane of midgut epithelial cells activates the insulin/insulin-like growth factor signaling (IIS) cascade leading to changes in mitochondrial activity. These can include changes in mitophagy, biogenesis, oxidative phosphorylation (OXPHOS) and the synthesis of nitric oxide (NO) and reactive oxygen species (ROS). These mitochondrial changes can in turn directly result in parasite killing and improve the integrity of the epithelial barrier, making it difficult for the parasite to escape from the midgut cell. In addition, mitochondrial changes in the midgut epithelium can influence other tissues, altering key physiologies such as reproduction, lifespan, metabolism and immunity that affect vectorial capacity.

of key physiologies in mosquitoes (Figure 1). When AMPK was overexpressed in the midgut of $D$. melanogaster, the age-related decline in epithelial barrier integrity was reduced, indicating that both autophagy and mitochondrial biogenesis protected the integrity of the midgut barrier (Rera et al., 2011; Ulgherait et al., 2014). In more recent work, treatment with rapamycin, which inhibits Akt-dependent mechanistic Target of Rapamycin (mTOR) signaling, maintained epithelial barrier integrity and extended lifespan in D. melanogaster independently of any changes in the gut microbiota (Schinaman et al., 2019). In mammals, AMPK is required for regulation of polarity of hepatocytes and other epithelial cell types as well as tight junction and barrier integrity through increased mitochondrial fusion and biogenesis (Treyer and Müsch, 2013; Kang et al., 2016; Ghosh, 2017). Successful $P$. falciparum infection of hepatocytes has been associated with reduced AMPK activity in these cells, whereas pharmacological induction of hepatocyte AMPK reduced intracellular parasite growth (Ruivo et al., 2016). In our work, we observed increased midgut barrier integrity in transgenic (TG) A. stephensi with increased PTEN expression in the midgut, which limited $P$. falciparum infection without changes to NF- $\mathrm{KB}$-dependent antiparasite gene expression (Hauck et al., 2013). Since the IIS activator Akt can suppress both mitochondrial biogenesis and autophagy, it is likely that overexpression of the IIS inhibitor PTEN in A. stephensi increased autophagy, mitochondrial fusion and mitochondrial biogenesis to enhance midgut barrier integrity, in turn increasing mosquito resistance to P. falciparum infection (Huang et al., 2011; Levine et al., 2011; Denton et al., 2012; Hauck et al., 2013). 
Supporting this idea, biomarker expression for both midgut stem cells (MSC) and autophagy were increased in TG A. stephensi overexpressing PTEN relative to non-transgenic (NTG) controls (Hauck et al., 2013). Collectively, our data and these observations suggest that manipulation of pathways leading to increased midgut mitochondrial fusion and biogenesis have the potential to improve midgut epithelial integrity and in turn resistance to $P$. falciparum infection in A. stephensi.

A proper balance between mitophagy and mitochondrial biogenesis is essential for optimal stem cell differentiation and maintenance. Previous studies have demonstrated the important roles stem cells play in the midgut of insects, including mosquitoes (Brown et al., 1985; Baton and Ranford-Cartwright, 2007). Intriguingly, Janeh et al. (2019) reported that, in contrast to Aedes albopictus and Culex pipiens, midgut tissue in A. gambiae does not contain cells that undergo mitosis to repair gut damage. The authors speculated that this difference could underlie permissiveness to malaria parasite infection and rightly suggested that additional mechanistic studies are necessary to fully understand the biological importance of these differences across mosquito genera. In $D$. melanogaster, upregulation of mitochondrial biogenesis in the midgut prevented MSC over-proliferation and the accumulation of improperly differentiated cells (Rera et al., 2012a). Coupled with an enhanced epithelial barrier, this phenotype confirmed that reduced MSC dysplasia could extend lifespan. When $A$. stephensi were provisioned with human IGF1 in the bloodmeal at concentrations consistent with titers found during malaria, midgut cytoprotection was enhanced through the homeostatic regulation of ROS and patterns of gene expression consistent with both MSC activity and autophagy, resulting in improved mitochondrial function and a more robust midgut epithelium (Drexler et al., 2014). These data suggested that enhancing mitochondrial function to increase midgut health could improve both parasite resistance and lifespan in A. stephensi (Drexler et al., 2014). In addition to these processes, the Hippo pathway regulates mitochondrial function in both human and D. melanogaster cells (Nagaraj et al., 2012). In D. melanogaster, knockdown of Hippo or overexpression of Yorkie, the transcriptional effector of the pathway, enhanced MSC proliferation and prevented apoptosis, providing additional support for the role of mitochondrial function in epithelial homeostasis (Huang et al., 2005; Ren et al., 2010).

\section{MITOCHONDRIAL FUNCTION CONTROLS DIVERSE PHYSIOLOGIES THAT ARE KEY TO VECTORIAL CAPACITY}

Host immunity to infection is regulated by mitochondrial function. Increased biogenesis of mitochondria during infection, possibly in response to cell injury, can alter the production of pro- and anti-inflammatory cytokines to improve mammalian host convalescence and survival (Sweeney et al., 2010; Carré et al., 2010; Sweeney et al., 2011; Tran et al., 2011; Dada and Sznajder, 2011; Piantadosi et al., 2011). ETC Complex I- and III-generated ROS can also activate Toll-like receptor signaling and NF-kB- dependent gene expression for the targeted elimination of pathogens (Arnoult et al., 2011). In A. stephensi, activation of IIS induced high levels of mitochondrial anti-parasite ROS and NO, responses that also resulted in significant damage to the midgut epithelium (Luckhart et al., 2013). This damage, however, can be mitigated by parasite-induced p38 MAPK signaling and associated increases in mitochondrial biogenesis, oxidative phosphorylation (OXPHOS) and antioxidant biosynthesis for mosquito survival during infection (Wang et al., 2015). In an effort to establish causeand-effect of these metabolic changes with infection outcome, we provisioned small molecule inhibitors to A. stephensi to demonstrate that midgut intermediary metabolism, in fact, regulates $P$. falciparum infection (Pietri et al., 2016). Notably, these small molecule inhibitors had no effect on NF-kBdependent immune gene expression, thereby linking anti-parasite resistance to shifts in glycolysis and mitochondrial function (Pietri et al., 2016). To our knowledge, this was the first evidence in anopheline mosquitoes that direct manipulation of metabolism and mitochondrial function could alter anti-parasite immunity.

Longevity is one of the most complex phenotypes regulated by mitochondrial physiology and is a life history trait intimately connected to resistance to infection across a wide range of organisms. Specifically, modest reductions in mitochondrial ETC activity and OXPHOS can enhance survival in organisms ranging from yeast to mice (reviewed in Rera et al., 2012a). A modest increase in ETC activity, however, can be effective as well. When ETC Complex I activity was increased in D. melanogaster through the overexpression of yeast $\mathrm{NADH}$-ubiquinone oxidoreductase the integrity of the midgut barrier was improved and lifespan extended, without a reduction in fecundity (Hur et al., 2013). The fact that both activation and inhibition of ETC Complex I can lead to increased longevity is an example of hormesis, where modest positive and negative changes can both drive an organism to improved mitochondrial function (Hur et al., 2014). Increased autophagy has also been found to extend lifespan of $D$. melanogaster. In this context, overexpression of Parkin, a key mediator of mitophagy, significantly extended lifespan and increased fruit fly fecundity (Rana et al., 2013). In contrast, mutations in PINK1, which binds to damaged mitochondria and recruits Parkin, were associated with a reduction in lifespan (Clark et al., 2006). In our work, PTEN overexpression in the A. stephensi midgut increased longevity, enhanced the midgut barrier, improved $P$. falciparum resistance and had no negative impact on fecundity (Hauck et al., 2013). This work suggested that PTEN overexpression in A. stephensi and the anticipated downstream effects on both PINK1 and Parkin improved mitochondrial quality control, which led to increased longevity and parasite resistance (Unoki and Nakamura, 2001). Extensive studies on AMPK in diverse organisms, including D. melanogaster and A. aegypti, demonstrated a key role for this protein in the regulation of senescence as well. In D. melanogaster, upregulation of AMPK in the midgut significantly extended lifespan (Ulgherait et al., 2014). In A. aegypti, a diet supplemented with polyphenols increased lifespan, in part through AMPK activation (Nunes et al., 2016). Our studies, however, did not replicate polyphenol-dependent life extension in A. stephensi, suggesting that these two mosquito species may have 
intriguing differences in longevity regulation (Johnson and Riehle, 2015).

Reproduction in mosquitoes, unlike most other Diptera including D. melanogaster, is intermittent and dependent on nutrient-rich bloodmeals, which initiate a reproductive cycle. This reproductive cycle, including oogenesis and vitellogenesis, is tightly regulated through a combination of hormonal signals, signaling cascades and nutrient sensors. In response to the blood-filled midgut, signals from the brain including insulin-like peptides (ILPs) and ovarian ecdysteroidogenic hormone (OEH) induce ovarian synthesis of 20-hydroxyecdysone (20E), which triggers fat body biosynthesis of lipids and other nutrients for egg development. The impact of $20 \mathrm{E}$ on malaria parasite infection is complex, with two studies reporting that $20 \mathrm{E}$ increases resistance of A. gambiae to P. falciparum and Plasmodium berghei (Upton et al., 2015; Reynolds et al., 2020). In contrast, Dahalan et al. (2019) showed that malederived 20E, delivered to the female mosquito during mating, dramatically altered the midgut transcriptome in patterns distinct from those of systemic effects of $20 \mathrm{E}$ and decreased resistance of Anopheles coluzzii to P. falciparum. In addition to IIS regulation of $20 \mathrm{E}$, IIS regulation of mitochondrial dynamics governs reproductive success in $D$. melanogaster while mitochondrial variation-dependent outcomes in innate immunity of fruit flies likely also drives immunity-fecundity tradeoffs. Notably, ubiquitous and neuron-specific overexpression of Parkin in adult fruit flies led to a significant increase in fecundity, in addition to lifespan extension (Rana et al., 2013). In a recent review, Salminen and Vale (2020) elegantly argue that variation in mitochondrial function of D. melanogaster, which has many hallmarks of the same biology in mammals, likely drives variation in fruit fly innate immune function. By extrapolation, this variation, which ranges from optimal mitochondrial dynamics to extreme dysfunction, would be predicted to underlie observed tradeoffs between immunity and fecundity observed in fruit flies with abnormal mitochondrial energy metabolism (Buchanan et al., 2018). Our own work in $A$. stephensi demonstrated that Akt signaling directly influences mitochondrial activity in the midgut (Luckhart et al., 2013). Further, increased Akt signaling in the fat body of A. stephensi, which most likely influences mitochondrial activity in this tissue as well, led to a significant increase in lifetime fecundity (Hun et al., 2019). In light of these observations, it is likely that IIS/TORdependent mitochondrial activity controls reproduction in Anopheles spp., but we would add that underlying IIS-dependent mitochondrial variation likely also drives the somewhat puzzling range of tradeoffs that are evident during infection.

\section{CONCLUSION}

The C. elegans and D. melanogaster guts are among the most stress sensitive tissues and function as critical "signaling centers" for communicating changes in mitochondrial function (Alic et al., 2014; Owusu-Ansah and Perrimon, 2015; Liu and Jin, 2017). For example, aging in D. melanogaster has been associated with a general decline in tissue mitochondrial biogenesis, but manipulations to systemically increase mitochondrial biogenesis had no effect on fruit fly longevity. However, targeted enhancements in gut-specific mitochondrial biogenesis enhanced D. melanogaster longevity via coordinated changes in tissue mitochondrial function (Vellai, 2009; Rera et al., 2012b; Ulgherait et al., 2014). Notably, gut mitochondrial biogenesis was associated with an improved midgut epithelial barrier, preventing lumenal microbes from initiating disseminated infections (Rera et al., 2012b). Accordingly, manipulation of gut mitochondrial function in D. melanogaster can enhance immunity locally and fitness systemically. Importantly, mitochondrial changes in fat body and muscle can contribute independently to these phenotypes as well, indicating that local mitochondrial changes induce functional systemic changes to extend local effects (Owusu-Ansah and Perrimon, 2015). Based on these observations, our data and the work of others, we hypothesize that changes in midgut mitochondrial dynamics function both locally and systemically to coordinate pathogen resistance, metabolism, lifespan and reproduction in Anopheles mosquitoes (Figure 1).

Is the Anopheles midgut a signaling center for mitochondrial physiology and the coordination of changes in host biology during infection? Published data and our data argue that this is case, but considerable work remains to be done in understanding this biology and its influence on mosquito resistance to infection, networking of life history traits critical to vectorial capacity and the dependency of this network on the coordination of mitochondrial function among the midgut and other tissues. Will this biology simply be a carbon copy of what is known from model invertebrates and mammals? If viewed only from the perspective of gene conservation, the answer is likely to be yes. However, the relevance of this biology to mosquitoes is uniquely elevated, defined and dictated by bloodfeeding and the consequences to human health that are vastly different from other organisms. Accordingly, this biology has both translational importance to "getting over the gut" and critical relevance to our basic scientific knowledge, with likely twists and turns that will provide a fascinating and substantive extension of our general knowledge of mitochondrial control of animal physiology.

\section{AUTHOR CONTRIBUTIONS}

SL and MR wrote and revised this mini-review. All authors contributed to the article and approved the submitted version.

\section{FUNDING}

The authors' work cited here has been supported by the National Institutes of Health, National Institute of Allergy and Infectious Diseases awards R01AI060664, R01AI080799, R56AI107263, R56AI118926, R56AI129420, R01AI073745, and R21AI125823.

\section{ACKNOWLEDGMENT}

We also acknowledge and appreciate many critical discussions of mosquito physiology over the course of our collective work with Dr. Mark R. Brown (University of Georgia). 


\section{REFERENCES}

Alic, N., Tullet, J. M., Niccoli, T., Broughton, S., Hoddinott, M. P., Slack, C., et al. (2014). Cell-nonautonomous effects of dFOXO/DAF-16 in aging. Cell Rep. 6, 608-616. doi: 10.1016/j.celrep.2014.01.015

Arik, A. J., Rasgon, J. L., Quicke, K. M., and Riehle, M. A. (2009). Manipulating insulin signaling to enhance mosquito reproduction. BMC Physiol. 9, 15. doi: 10.1186/1472-6793-9-15

Arik, A. J., Hun, L. V., Quicke, K., Piatt, M., Ziegler, R., Scaraffia, P. Y., et al. (2015). Increased Akt signaling in the mosquito fat body increases adult survivorship. FASEB J. 29, 1404-1413. doi: 10.1096/fj.14-261479

Arnoult, D., Soares, F., Tattoli, I., and Girardin, S. E. (2011). Mitochondria in innate immunity. EMBO Rep. 12, 901-910. doi: 10.1038/embor.2011.157

Baton, L., and Ranford-Cartwright, L. (2007). Morphological evidence for proliferative regeneration of the Anopheles stephensi midgut epithelium following Plasmodium falciparum ookinete invasion. J. Invertebr. Pathol. 96, 244-254. doi: 10.1016/j.jip.2007.05.005

Brown, M. R., Raikhel, A. S., and Lea, A. O. (1985). Ultrastructure of midgut endocrine cells in the adult mosquito, Aedes aegypti. Tissue Cell. 17, 709-721. doi: 10.1016/0040-8166(85)90006-0

Brown, M. R., Clark, K. D., Gulia, M., Zhao, Z., Garczynski, S. F., Crim, J. W., et al. (2008). An insulin-like peptide regulates egg maturation and metabolism in the mosquito Aedes aegypti. Proc. Natl. Acad. Sci. U. S. A. 105, 5716-5721. doi: $10.1073 /$ pnas.0800478105

Buchanan, J. L., Meiklejohn, C. D., and Montooth, K. L. (2018). Mitochondrial dysfunction and infection generate immunity-fecundity tradeoffs in Drosophila. Integr. Comp. Biol. 58, 591-603. doi: 10.1093/icb/icy078

Carré, J. E., Orban, J. C., Re, L., Felsmann, K., Iffert, W., Bauer, M., et al. (2010). Survival in critical illness is associated with early activation of mitochondrial biogenesis. Am. J. Respir. Crit. Care Med. 182, 745-751. doi: 10.1164/rccm.201003-0326OC

Charmpilas, N., and Tavernarakis, N. (2020). Mitochondrial maturation drives germline stem cell differentiation in Caenorhabditis elegans. Cell Death Differ. 27, 601-617. doi: 10.1038/s41418-019-0375-9

Chaudhari, S. N., and Kipreos, E. T. (2017). Increased mitochondrial fusion allows the survival of older animals in diverse C. elegans longevity pathways. Nat. Commun. 8, 1-16. doi: 10.1038/s41467-017-00274-4

Cheng, Z., Guo, S., Copps, K., Dong, X., Kollipara, R., Rodgers, J. T., et al. (2009). Foxol integrates insulin signaling with mitochondrial function in the liver. Nat. Med. 15, 1307-1311. doi: 10.1038/nm.2049

Cheng, Z., Tseng, Y., and White, M. F. (2010). Insulin signaling meets mitochondria in metabolism. Trends Endrocrinol. Metab. 21, 589-598. doi: 10.1016/j.tem.2010.06.005

Chikka, M. R., Anbalagan, C., Dvorak, K., Dombeck, K., and Prahlad, V. (2016). The mitochondria-regulated immune pathway activated in the C. elegans intestine is neuroprotective. Cell Rep. 16, 2399-2414. doi: 10.1016/ j.celrep.2016.07.077

Clark, I. E., Dodson, M. W., Jiang, C., Cao, J. H., Huh, J. R., Seol, J. H., et al. (2006). Drosophila pink1 is required for mitochondrial function and interacts genetically with parkin. Nature 441, 1162-1166. doi: 10.1038/nature04779

Corby-Harris, V., Drexler, A., Watkins de Jong, L., Antonova, Y., Pakpour, N., Ziegler, R., et al. (2010). Activation of Akt signaling reduces the prevalence and intensity of malaria parasite infection and lifespan in Anopheles stephensi mosquitoes. PLoS Pathog. 6, e1001003. doi: 10.1371/journal.ppat.1001003

Corona Velazquez, A. F., and Jackson, W. T. (2018). So many roads: The multifaceted regulation of autophagy induction. Mol. Cell. Biol. 38, e00303e00318. doi: 10.1128/MCB.00303-18

Dada, L. A., and Sznajder, J. II (2011). Mitochondrial Ca2 and ROS take center stage to orchestrate TNF- $\alpha$-mediated inflammatory responses. J. Clin. Invest. 121, 1683-1685. doi: 10.1172/JCI57748

Dahalan, F. A., Churcher, T. S., Windbichler, N., and Lawniczak, M. K. (2019). The male mosquito contribution towards malaria transmission: Mating influences the Anopheles female midgut transcriptome and increases female susceptibility to human malaria parasites. PLoS Pathog. 15, e1008063. doi: 10.1371/journal.ppat.1008063

Denton, D., Chang, T., Nicolson, S., Shravage, B., Simin, R., Baehrecke, E., et al. (2012). Relationship between growth arrest and autophagy in midgut programmed cell death in Drosophila. Cell Death Differ. 19, 1299-1307. doi: $10.1038 / \mathrm{cdd} .2012 .43$
Drexler, A. L., Pietri, J. E., Pakpour, N., Hauck, E., Wang, B., Glennon, E. K., et al. (2014). Human IGF1 regulates midgut oxidative stress and epithelial homeostasis to balance lifespan and Plasmodium falciparum resistance in Anopheles stephensi. PLoS Pathog. 10, e1004231. doi: 10.1371/journal.ppat.1004231

Elgass, K., Pakay, J., Ryan, M. T., and Palmer, C. S. (2012). Recent advances into the understanding of mitochondrial fission. BBA-Mol. Cell. Res. 1833, 150-161. doi: 10.1016/j.bbamcr.2012.05.002

Fernandez-Marcos, P. J., and Auwerx, J. (2011). Regulation of PGC-1 $\alpha$, a nodal regulator of mitochondrial biogenesis. Am. J. Clin. Nutr. 93, 884S-890S. doi: 10.3945/ajcn.110.001917

Garcia-Cao, I., Song, M. S., Hobbs, R. M., Laurent, G., Giorgi, C., de Boer, V. C. J., et al. (2012). Systemic elevation of PTEN induces a tumor-suppressive metabolic state. Cell 149, 49-62. doi: 10.1016/j.cell.2012.02.030

Ghosh, P. (2017). The stress polarity pathway: AMPK 'GIV'-es protection against metabolic insults. Aging 9, 303-314. doi: 10.18632/aging.101179

Graf, R., Neuenschwander, S., Brown, M. R., and Ackermann, U. (1997). Insulinmediated secretion of ecdysteroids from mosquito ovaries and molecular cloning of the insulin receptor homologue from ovaries of bloodfed Aedes aegypti. Insect Mol. Biol. 6, 151-163. doi: 10.1111/j.1365-2583.1997.tb00083.x

Gulia-Nuss, M., Robertson, A. E., Brown, M. R., and Strand, M. R. (2011). Insulinlike peptides and the target of rapamycin pathway coordinately regulate blood digestion and egg maturation in the mosquito Aedes aegypti. PLoS One 6, e20401. doi: 10.1371/journal.pone.0020401

Hauck, E. S., Antonova-Koch, Y., Drexler, A., Pietri, J., Pakpour, N., Liu, D., et al. (2013). Overexpression of phosphatase and tensin homolog improves fitness and decreases Plasmodium falciparum development in Anopheles stephensi. Microbes Infect. 15, 775-787. doi: 10.1016/j.micinf.2013.05.006

Horton, A. A., Lee, Y., Coulibaly, C. A., Rashbrook, V. K., Cornel, A. J., Lanzaro, G. C., et al. (2010). Identification of three single nucleotide polymorphisms in Anopheles gambiae immune signaling genes that are associated with natural Plasmodium falciparum infection. Malar. J. 9, 160. doi: 10.1186/1475-2875-9-160

Huang, J., Wu, S., Barrera, J., Matthews, K., and Pan, D. (2005). The Hippo signaling pathway coordinately regulates cell proliferation and apoptosis by inactivating yorkie, the Drosophila homolog of YAP. Cell 122, 421-434. doi: 10.1016/j.cell.2005.06.007

Huang, J., Lam, G. Y., and Brumell, J. H. (2011). Autophagy signaling through reactive oxygen species. Antioxid. Redox Signal. 14, 2215-2231. doi: 10.1089/ ars. 2010.3554

Hun, L. V., Luckhart, S., and Riehle, M. A. (2019). Increased Akt signaling in the fat body of Anopheles stephensi extends lifespan and increases lifetime fecundity through modulation of insulin-like peptides. J. Insect Physiol. 118, 103932. doi: 10.1016/j.jinsphys.2019.103932

Hur, J. H., Bahadorani, S., Graniel, J., Koehler, C. L., Ulgherait, M., Rera, M., et al. (2013). Increased longevity mediated by yeast NDI1 expression in Drosophila intestinal stem and progenitor cells. Aging (Albany NY) 5, 662-681. doi: 10.18632/aging.100595

Hur, J. H., Stork, D. A., and Walker, D. W. (2014). Complex-I-ty in aging. J. Bioenerg. Biomembr. 46, 329-335. doi: 10.1007/s10863-014-9553-0

Hur, L. V., Luckhart, S., and Riehle, M. A. (2019). Increased Akt signaling in the fat body of Anopheles stephensi extends lifespan and increases lifetime fecundity through modulation of insulin-like peptides. J. Insect Physiol. 118, 103932. doi: 10.1016/j.jinsphys.2019.103932

Janeh, M., Osman, D., and Kambris, Z. (2019). Comparative analysis of midgut regeneration capacity and resistance to oral infection in three disease-vector mosquitoes. Sci. Rep. 9, 1-10. doi: 10.1038/s41598-019-50994-4

Johnson, A. A., and Riehle, M. A. (2015). Resveratrol fails to extend life span in the mosquito Anopheles stephensi. Rejuvenation Res. 18, 473-478. doi: 10.1089/ rej.2015.1670

Kang, C., You, Y. J., and Avery, L. (2007). Dual roles of autophagy in the survival of Caenorhabditis elegans during starvation. Genes Dev. 21, 2161-2171. doi: $10.1101 /$ gad.1573107

Kang, S. W. S., Haydar, G., Taniane, C., Farrell, G., Arias, I. M., Lippincott-Schwartz, J., et al. (2016). AMPK activation prevents and reverses drug-induced mitochondrial and hepatocyte injury by promoting mitochondrial fusion and function. PloS One 11, e0165638. doi: 10.1371/journal.pone.0165638

Kwon, S., Kim, E. J. E., and Lee, S. V. (2018). Mitochondria-mediated defense mechanisms against pathogens in Caenorhabditis elegans. BMB Rep. 51, 274279. doi: 10.5483/BMBRep.2018.51.6.111 
Levine, B., Mizushima, N., and Virgin, H. W. (2011). Autophagy in immunity and inflammation. Nature 469, 323-335. doi: 10.1038/nature09782

Lim, J., Gowda, D. C., Krishnegowda, G., and Luckhart, S. (2005). Induction of nitric oxide synthase in Anopheles stephensi by Plasmodium falciparum: mechanism of signaling and the role of parasite glycosylphosphatidylinositols. Infect. Immun. 73, 2778-2789. doi: 10.1128/IAI.73.5.2778-2789.2005

Liu, Q., and Jin, L. H. (2017). Organ-to-organ communication: a Drosophila gastrointestinal tract perspective. Front. Cell Dev. Biol. 5, 29. doi: 10.3389/ fcell.2017.00029

Luckhart, S., Giulivi, C., Drexler, A. L., Antonova-Koch, Y., Sakaguchi, D., Napoli, E., et al. (2013). Sustained activation of Akt elicits mitochondrial dysfunction to block Plasmodium falciparum infection in the mosquito host. PLoS Pathog. 9, e1003180. doi: 10.1371/journal.ppat.1003180

Luckhart, S., and Riehle, M. A. (2017). "Conservation and convergence of immune signaling pathways with mitochondrial regulation in vector arthropod physiology," in Arthropod Vector: Controller of Disease Transmission, Volume 1 (London, United Kingdom: Elsevier), 15-33. doi: 10.1016/B978-012-805350-8.00002-7

Ma, K., Chen, G., Li, W., Kepp, O., Zhu, Y., and Chen, Q. (2020). Mitophagy, mitochondrial homeostasis, and cell fate. Front. Cell Dev. Biol. 8, 467. doi: 10.3389 /fcell.2020.00467

Manning, B. D., and Toker, A. (2017). AKT/PKB signaling: navigating the network. Cell 169, 381-405. doi: 10.1016/j.cell.2017.04.001

Marquez, A. G., Pietri, J. E., Smithers, H. M., Nuss, A., Antonova, Y., Drexler, A. L., et al. (2011). Insulin-like peptides in the mosquito Anopheles stephensi: identification and expression in response to diet and infection with Plasmodium falciparum. Gen. Comp. Endocrinol. 173, 303-312. doi: 10.1016/ j.ygcen.2011.06.005

Miguel-Aliaga, I., Jasper, H., and Lemaitre, B. (2018). Anatomy and physiology of the digestive tract of Drosophila melanogaster. Genetics 210, 357-396. doi: 10.1534/genetics.118.300224

Mukherjee, S., Basar, M. A., Davis, C., and Duttaroy, A. (2014). Emerging functional similarities and divergences between Drosophila Spargel/dPGC-1 and mammalian PGC-1 protein. Front. Genet. 5, 216. doi: 10.3389/fgene. 2014.00216

Nagaraj, R., Gururaja-Rao, S., Jones, K. T., Slattery, M., Negre, N., Braas, D., et al. (2012). Control of mitochondrial structure and function by the Yorkie/YAP oncogenic pathway. Genes Dev. 26, 2027-2037. doi: 10.1101/gad.183061.111

Nisoli, E., Clementi, E., Paolucci, C., Cozzi, V., Tonello, C., Sciorati, C., et al. (2003). Mitochondrial biogenesis in mammals: the role of endogenous nitric oxide. Sci. Signal. 299, 896-899. doi: 10.1126/science.1079368

Nisoli, E., Falcone, S., Tonello, C., Cozzi, V., Palomba, L., Fiorani, M., et al. (2004). Mitochondrial biogenesis by NO yields functionally active mitochondria in mammals. Proc. Natl. Acad. Sci. U. S. A. 101, 16507-16512. doi: 10.1073/ pnas.0405432101

Nisoli, E., Tonello, C., Cardile, A., Cozzi, V., Bracale, R., Tedesco, L., et al. (2005). Calorie restriction promotes mitochondrial biogenesis by inducing the expression of eNOS. Sci. Signal. 310, 314-317. doi: 10.1126/science.1117728

Nunes, R. D., Ventura-Martins, G., Moretti, D. M., Medeiros-Castro, P., RochaSantos, C., de Oliveira, D.-F., et al. (2016). Polyphenol-rich diets exacerbate ampk-mediated autophagy, decreasing proliferation of mosquito midgut microbiota, and extending vector lifespan. PLoS Negl. Trop. Dis. 10, e0005034. doi: 10.1371/journal.pntd.0005034

Nuss, A. B., and Brown, M. R. (2018). Isolation of an insulin-like peptide from the Asian malaria mosquito, Anopheles stephensi, that acts as a steroidogenic gonadotropin across diverse mosquito taxa. Gen. Comp. Endocrinol. 258, 140148. doi: 10.1016/j.ygcen.2017.05.007

Owusu-Ansah, E., and Perrimon, N. (2015). Stress signaling between organs in Metazoa. Annu. Rev. Cell Dev. Biol. 31, 497-522. doi: 10.1146/annurev-cellbio100814-125523

Pakpour, N., Corby-Harris, V., Green, G. P., Smithers, H. M., Cheung, K. W., Riehle, M. A., et al. (2012). Ingested human insulin inhibits the mosquito NFkappaB-dependent immune response to Plasmodium falciparum. Infect. Immun. 80, 2141-2149. doi: 10.1128/IAI.00024-12

Pakpour, N., Camp, L., Smithers, H. M., Wang, B., Tu, Z., Nadler, S. A., et al. (2013). Protein Kinase C-dependent signaling controls the midgut epithelial barrier to malaria parasite infection in Anopheline mosquitoes. PLoS One 8, e76535. doi: 10.1371/journal.pone.0076535
Palikaras, K., Lionaki, E., and Tavernarakis, N. (2018). Mechanisms of mitophagy in cellular homeostasis, physiology and pathology. Nat. Cell Biol. 20, $1013-$ 1022. doi: $10.1038 / s 41556-018-0176-2$

Piantadosi, C. A., Withers, C. M., Bartz, R. R., MacGarvey, N. C., Fu, P., Sweeney, T. E., et al. (2011). Heme oxygenase-1 couples activation of mitochondrial biogenesis to anti-inflammatory cytokine expression. J. Biol. Chem. 286, 16374-16385. doi: 10.1074/jbc.M110.207738

Pietri, J. E., Pakpour, N., Napoli, E., Song, G., Pietri, E., Potts, R., et al. (2016). Two insulin-like peptides differentially regulate malaria parasite infection in the mosquito through effects on intermediary metabolism. Biochem. J. 437, 34873503. doi: 10.1042/BCJ20160271

Rana, A., Rera, M., and Walker, D. W. (2013). Parkin overexpression during aging reduces proteotoxicity, alters mitochondrial dynamics, and extends lifespan. Proc. Natl. Acad. Sci. U. S. A. 110, 8638-8643. doi: 10.1073/pnas.1216197110

Ren, F., Wang, B., Yue, T., Yun, E. Y., Ip, Y. T., and Jiang, J. (2010). Hippo signaling regulates Drosophila intestine stem cell proliferation through multiple pathways. Proc. Natl. Acad. Sci. U. S. A. 107, 21064-21069. doi: $10.1073 /$ pnas. 1012759107

Rera, M., Bahadorani, S., Cho, J., Koehler, C. L., Ulgherait, M., Hur, J. H., et al. (2011). Modulation of longevity and tissue homeostasis by the Drosophila PGC-1 homolog. Cell Metab. 14, 623-634. doi: 10.1016/j.cmet.2011.09.013

Rera, M., Azizi, M. J., and Walker, D. W. (2012a). Organ-specific mediation of lifespan extension: More than a gut feeling? Ageing Res. Rev. 12, 436-444. doi: 10.1016/j.arr.2012.05.003

Rera, M., Clark, R.II, and Walker, D. W. (2012b). Intestinal barrier dysfunction links metabolic and inflammatory markers of aging to death in Drosophila. Proc. Natl. Acad. Sci. U. S. A. 109, 21528-21533. doi: 10.1073/pnas. 1215849110

Reynolds, R. A., Kwon, H., and Smith, R. C. (2020). 20-Hydroxyecdysone primes innate immune responses that limit bacterial and malarial parasite survival in Anopheles gambiae. Msphere 5, 10.1128/mSphere.00983-19. doi: 10.1128/ mSphere.00983-19

Riehle, M. A., and Brown, M. R. (1999). Insulin stimulates ecdysteroid production through a conserved signaling cascade in the mosquito Aedes aegypti. Insect Biochem. Mol. Biol. 29, 855-860. doi: 10.1016/S0965-1748 (99)00084-3

Riehle, M. A., and Brown, M. R. (2002). Insulin receptor expression during development and a reproductive cycle in the ovary of the mosquito Aedes aegypti. Cell Tissue Res. 308, 409-420. doi: 10.1007/s00441-002-0561-8

Riehle, M. A., and Brown, M. R. (2003). Molecular analysis of the serine/threonine kinase Akt and its expression in the mosquito Aedes aegypti. Insect Mol. Biol. 12, 225-232. doi: 10.1046/j.1365-2583.2003.00405.x

Roy, S. G., Hansen, I. A., and Raikhel, A. S. (2007). Effect of insulin and 20hydroxyecdysone in the fat body of the yellow fever mosquito, Aedes aegypti. Insect Biochem. Mol. Biol. 37, 1317-1326. doi: 10.1016/j.ibmb.2007.08.004

Ruegsegger, G. N., Creo, A. L., Cortes, T. M., Dasari, S., and Nair, K. S. (2018). Altered mitochondrial function in insulin-deficient and insulin-resistant states. J. Clin. Invest. 128, 3671-3681. doi: 10.1172/JCI120843

Ruivo, M. T. G., Vera, I. M., Sales-Dias, J., Meireles, P., Gural, N., Bhatia, S. N., et al. (2016). Host AMPK is a modulator of Plasmodium liver infection. Cell Rep. 16, 2539-2545. doi: 10.1016/j.celrep.2016.08.001

Sadagurski, M., and White, M. F. (2013). Integrating metabolism and longevity through insulin and IGF1 signaling. Endocrinol. Metab. Clin. North Am. 42, 127-148. doi: 10.1016/j.ecl.2012.11.008

Salminen, T. S., and Vale, P. F. (2020). Drosophila as a model system to investigate the effects of mitochondrial variation on innate immunity. Front. Immunol. 11:521. doi: 10.3389/fimmu.2020.00521

Schinaman, J. M., Rana, A., William, W. J., Clark, R.II, and Walker, D. W. (2019). Rapamycin modulates tissue aging and lifespan independently of the gut microbiota in Drosophila. Sci. Rep. 9, 1-10. doi: 10.1038/s41598-019-44106-5

Sheard, K. M., Thibault-Sennett, S. A., Sen, A., Shewmaker, F., and Cox, R. T. (2020). Clueless forms dynamic, insulin-responsive bliss particles sensitive to stress. Dev. Biol. 459, 149-160. doi: 10.1016/j.ydbio.2019.12.004

Sim, C., and Denlinger, D. L. (2008). Insulin signaling and FOXO regulate the overwintering diapause of the mosquito Culex pipiens. Proc. Natl. Acad. Sci. U. S. A. 105, 6777-6781. doi: 10.1073/pnas.0802067105

Surachetpong, W., Pakpour, N., Cheung, K. W., and Luckhart, S. (2011). Reactive oxygen species-dependent cell signaling regulates the mosquito immune 
response to Plasmodium falciparum. Antioxid. Redox Signal. 14, 943-955. doi: 10.1089/ars.2010.3401

Sweeney, T. E., Suliman, H. B., Hollingsworth, J. W., and Piantadosi, C. A. (2010). Differential regulation of the PGC family of genes in a mouse model of Staphylococcus aureus sepsis. PLoS One 5, e11606. doi: 10.1371/ journal.pone.0011606

Sweeney, T. E., Suliman, H. B., Hollingsworth, J. W., Welty-Wolf, K. E., and Piantadosi, C. A. (2011). A Toll-like receptor 2 pathway regulates the Ppargcla/b metabolic co-activators in mice with Staphylococcal aureus sepsis. PLoS One 6, e25249. doi: 10.1371/journal.pone.0025249

Tiefenbock, S. K., Baltzer, C., Egli, N. A., and Frei, C. (2010). The Drosophila PGC1 homologue Spargel coordinates mitochondrial activity to insulin signalling. EMBO J. 29, 171-183. doi: 10.1038/emboj.2009.330

Tóth, M. L., Sigmond, T., Borsos, É, Barna, J., Erdélyi, P., Takács-Vellai, K., et al. (2008). Longevity pathways converge on autophagy genes to regulate life span in Caenorhabditis elegans. Autophagy 4, 330-338. doi: 10.4161/auto.5618

Tran, M., Tam, D., Bardia, A., Bhasin, M., Rowe, G. C., Kher, A., et al. (2011). PGC- $1 \alpha$ promotes recovery after acute kidney injury during systemic inflammation in mice. J. Clin. Invest. 121, 4003-4014. doi: 10.1172/JCI58662

Treyer, A., and Müsch, A. (2013). Hepatocyte Polarity. Compr. Physiol. 3, 243-287 doi: 10.1002/cphy.c120009

Ulgherait, M., Rana, A., Rera, M., Graniel, J., and Walker, D. W. (2014). AMPK modulates tissue and organismal aging in a non-cell-autonomous manner. Cell Rep. 8, 1767-1780. doi: 10.1016/j.celrep.2014.08.006

Unoki, M., and Nakamura, Y. (2001). Growth-suppressive effects of BPOZ and EGR2, two genes involved in the PTEN signaling pathway. Oncogene 20, 44574465. doi: 10.1038/sj.onc. 1204608

Upton, L. M., Povelones, M., and Christophides, G. K. (2015). Anopheles gambiae blood feeding initiates an anticipatory defense response to Plasmodium berghei. J. Innate. Immun. 7, 74-86. doi: 10.1159/000365331

Vellai, T. (2009). Autophagy genes and ageing. Cell Death Differ. 16, 94-102. doi: $10.1038 /$ cdd. 2008.126
Wang, B., Pakpour, N., Napoli, E., Drexler, A., Glennon, E., Surachetpong, W., et al. (2015). Anopheles stephensi p38 MAPK signaling induces tolerance during Plasmodium falciparum infection. Parasit. Vectors 8, 424. doi: 10.1186/s13071-015-1016-x

Wang, Z., Liu, Y., Chaitankar, V., Pirooznia, M., and Xu, H. (2019). Electron transport chain biogenesis activated by a JNK-insulin-Myc relay primes mitochondrial inheritance in Drosophila. Elife 8, e49309. doi: 10.7554/ eLife.49309.043

Wardelmann, K., Blümel, S., Rath, M., Alfine, E., Chudoba, C., Schell, M., et al. (2019). Insulin action in the brain regulates mitochondrial stress responses and reduces diet-induced weight gain. Parasit. Vectors 21, 68-81. doi: 10.1016/ j.molmet.2019.01.001

Youle, R. J., and van der Bliek, A. M. (2012). Mitochondrial fission, fusion, and stress. Science 337, 1062-1065. doi: 10.1126/science.1219855

Yu, R., Lendahl, U., Nister, M., and Zhao, J. (2020). Regulation of mammalian mitochondrial dynamics: Opportunities and challenges. Front. Endocrinol. 11, 374. doi: 10.3389/fendo.2020.00374

Zhu, J., Wang, K. Z., and Chu, C. T. (2013). After the banquet: mitochondrial biogenesis, mitophagy, and cell survival. Autophagy 9, 1663-1676. doi: 10.4161 /auto. 24135

Conflict of Interest: The authors declare that the research was conducted in the absence of any commercial or financial relationships that could be construed as a potential conflict of interest.

Copyright (C) 2020 Luckhart and Riehle. This is an open-access article distributed under the terms of the Creative Commons Attribution License (CC BY). The use, distribution or reproduction in other forums is permitted, provided the original author(s) and the copyright owner(s) are credited and that the original publication in this journal is cited, in accordance with accepted academic practice. No use, distribution or reproduction is permitted which does not comply with these terms. 